Case Report

\title{
Immune Complex Mediated Glomerulonephritis with Acute Thrombotic Microangiopathy following Newly Detected Hepatitis B Virus Infection in a Kidney Transplant Recipient
}

\author{
Tracey Salter, ${ }^{1}$ Hannah Burton, ${ }^{1}$ Sam Douthwaite, ${ }^{2}$ William Newsholme, ${ }^{3}$ \\ Catherine Horsfield, ${ }^{4}$ and Rachel Hilton ${ }^{1}$ \\ ${ }^{1}$ Departments of Nephrology and Transplantation, Guy's and St Thomas' NHS Foundation Trust, London, UK \\ ${ }^{2}$ Department of Virology, Guy's and St Thomas' NHS Foundation Trust, London, UK \\ ${ }^{3}$ Department of Infectious Diseases, Guy's and St Thomas' NHS Foundation Trust, London, UK \\ ${ }^{4}$ Department of Histopathology, Guy's and St Thomas' NHS Foundation Trust, London, UK \\ Correspondence should be addressed to Tracey Salter; traceysalter@doctors.org.uk
}

Received 5 June 2016; Accepted 8 September 2016

Academic Editor: David Conti

Copyright (c) 2016 Tracey Salter et al. This is an open access article distributed under the Creative Commons Attribution License, which permits unrestricted use, distribution, and reproduction in any medium, provided the original work is properly cited.

\begin{abstract}
Hepatitis B virus (HBV) presents a risk to patients and staff in renal units. To minimise viral transmission, there are international and UK guidelines recommending HBV immunisation for patients commencing renal replacement therapy (RRT) and HBV surveillance in kidney transplant recipients. We report the case of a 56-year-old male who was immunised against HBV before starting haemodialysis. He received a deceased donor kidney transplant three years later, at which time there was no evidence of HBV infection. After a further six years he developed an acute kidney injury; allograft biopsy revealed an acute thrombotic microangiopathy (TMA) with glomerulitis, peritubular capillaritis, and C4d staining. Due to a "full house" immunoprofile, tests including virological screening were undertaken, which revealed acute HBV infection. Entecavir treatment resulted in an improvement in viral load and kidney function. HBV genotyping demonstrated a vaccine escape mutant, suggesting "past resolved" infection that reactivated with immunosuppression, though posttransplant acquisition cannot be excluded. This is the first reported case of acute HBV infection associated with immune complex mediated glomerulonephritis and TMA. Furthermore, it highlights the importance of HBV surveillance in kidney transplant recipients, which although addressed by UK guidelines is not currently practiced in all UK units.
\end{abstract}

\section{Introduction}

Blood borne virus (BBV) infection presents a risk to both patients and staff in renal centres, with HBV historically associated with outbreaks in haemodialysis units. Globally there exist numerous clinical practice guidelines (CPG) which aim to minimise viral transmission. International CPG recommend that patients who require RRT should be immunised against HBV $[1,2]$. There is specific guidance for HBV surveillance in patients receiving regular hospital haemodialysis; those deemed to have achieved protective immunity (anti-hepatitis B surface antibody [anti-HBs] $>10 \mathrm{mIU} / \mathrm{mL}$ ) need to only be tested for hepatitis B surface antigen ( $\mathrm{HBsAg}$ ) annually [1, 2]. In addition, there is international [3] and UK [4] guidance addressing HBV surveillance in kidney transplant recipients.

TMA is characterised by microangiopathic haemolytic anaemia, thrombocytopenia associated with hyaline thrombi, and varying degrees of end organ failure. Numerous and varied causes are recognised, including viral infection [5]. However, there are no reports of TMA in association with acute HBV infection. Here we describe the case of a kidney transplant recipient who developed TMA synchronous to newly detected HBV infection in the absence of other likely causes. 


\section{Case Report}

A fifty-six-year-old Arab male developed end stage kidney disease (ESKD) in association with hypertension and type 2 diabetes mellitus and commenced unit haemodialysis. At the time his serology tested negative for HBsAg and HBV core antibody $(\mathrm{cAb})$. In accordance with UK guidance he received a full course of vaccination against $\mathrm{HBV}$ infection, receiving HBVaxPro $10 \mu \mathrm{g}$ at 0,1 , and 6 months; subsequent anti-HBs titre following completion of immunisation was $965 \mathrm{mIU} / \mathrm{mL}$. He was therefore deemed to have acquired protective immunity and was scheduled for annual monitoring of anti-HBs levels, with a booster indicated for any level $<100 \mathrm{mIU} / \mathrm{mL}$. One year after immunisation the anti-HBs level was $155 \mathrm{mIU} / \mathrm{mL}$, suggesting that protective immunity had been maintained and that no booster was required.

Three years after starting dialysis he received a donation after cardiac death (DCD) deceased donor kidney transplant. At the time he remained $\mathrm{HBsAg}$ and $\mathrm{cAb}$ negative. Induction immunosuppression comprised basiliximab and methylprednisolone. Maintenance immunosuppression was ciclosporin (target level 200-300 $\mathrm{g} / \mathrm{L}$ ), mycophenolate mofetil $500 \mathrm{mg}$ four times daily, and corticosteroids. The postoperative course was complicated by delayed graft function and T cellmediated rejection requiring treatment with pulsed methylprednisolone and antithymocyte globulin (ATG) (Figure 1(a)). Cytomegalovirus (CMV) viraemia was detected and treated with valganciclovir. Thereafter his kidney function improved to a baseline serum creatinine of $130 \mu \mathrm{mol} / \mathrm{L}$. Maintenance immunosuppression comprised twice-daily tacrolimus with levels maintained between 7 and $12 \mu \mathrm{g} / \mathrm{L}$, mycophenolate mofetil $500 \mathrm{mg}$ twice daily, and prednisolone $5 \mathrm{mg}$ daily.

Over the next five years his allograft function remained stable. Two immunosuppression-related issues arose: Escherichia coli sepsis secondary to cellulitis and a pyogenic granuloma of his thumb. In line with current practice at our renal unit, anti-HBs levels were not checked and no boosters were administered after transplant.

Six years after transplantation he presented to his local hospital nonspecifically unwell, and was found to have acute kidney injury (AKI) with a rise in creatinine from a baseline of $100 \mu \mathrm{mol} / \mathrm{L}$ to almost $350 \mu \mathrm{mol} / \mathrm{L}$. He was transferred to this hospital for further management (Figure 1(b)).

Allograft biopsy revealed TMA with glomerulitis (g3), mild peritubular capillaritis (ptc1), and acute tubular injury, together with $\mathrm{C} 4 \mathrm{~d}$ staining $(\mathrm{C} 4 \mathrm{~d} 2)$ (Figure 2). This was attributed to antibody-mediated rejection, although no donor-specific antibody was detected in peripheral blood, and he commenced treatment with pulsed intravenous methylprednisolone (3x $500 \mathrm{mg}$ ), plasma exchange (4-litre exchanges), and intravenous immunoglobulin $(400 \mathrm{mg} / \mathrm{kg})$. Serum creatinine remained significantly raised at $255 \mu \mathrm{mol} / \mathrm{L}$ (Figure 1(b)).

As a possible cause for TMA, tacrolimus was stopped. Immunosuppression then comprised mycophenolate mofetil $500 \mathrm{mg}$ twice daily and prednisolone $5 \mathrm{mg}$ daily.

The biopsy was further examined, and, in light of the extent of the glomerular thrombotic process and endothelial hypercellularity, a native immunoperoxidase panel was performed. This revealed a "full house" immunoprofile ( $\operatorname{IgA}++$, $\mathrm{IgG}++, \operatorname{IgM}++, \mathrm{C} 3++$, and $\mathrm{Clq}++$ ) within the glomeruli, with deposition in the mesangial matrix and also at subendothelial loci (Figure 2), consistent with an immune complex mediated glomerulonephritis. This finding triggered both virological and lupus screening.

Initial $\mathrm{HBV}$ testing revealed the following: $\mathrm{HBsAg}$ detected; hepatitis B e antigen detected; hepatitis B e antibody not detected; hepatitis B core IgM not detected; HBV viral load (Roche) HBV DNA log value 8.15. Serology was negative for the following: CMV, BK virus, Epstein-Barr virus, HIV, hepatitis $\mathrm{C}$ virus, delta virus, and parvovirus. Lupus and antiphospholipid screening tests were negative, as was $E$. coli 0157 serology. All previous tests for HBsAg had been negative, most recently at the time of transplantation.

Liver function tests, including markers of synthetic function, were normal. He commenced treatment with Entecavir and completed ten plasma exchanges for ongoing TMA with thrombocytopenia.

The source of his HBV infection was sought. He denied any risk factors. His immediate contacts all screened negative for $\mathrm{HBV}$ infection. The allograft donor was $\mathrm{HBsAg}$ and $\mathrm{cAb}$ negative. Hepatitis $B$ genotyping showed a vaccine escape mutant. One possibility is that the $\mathrm{HBV}$ cAb result at the time of starting dialysis was a false negative (commercial assays for the detection of $\mathrm{HBV} \mathrm{cAb}$ can show marked variability in detecting $\mathrm{cAb}$ in comparative studies). He could thus have had resolved $\mathrm{HBV}$ infection prior to transplantation which later reactivated, with $\mathrm{HBV} \mathrm{sAb}$ driving viral mutation. Another possibility is new acquisition of mutated $\mathrm{HBV}$, against which the vaccine-induced sAb was not protective. Revisiting his history revealed that he had travelled to Mecca in the months prior to his presentation with AKI; it is possible that HBV could have been acquired during ritual headshaving.

A repeat allograft biopsy was performed after two weeks of Entecavir treatment. This showed resolution of the TMA, but with residual immune complex mediated glomerulonephritis.

After one month of Entecavir treatment the HBV viral load had dropped from a log value of 8.15 to a log value of 4.65; the creatinine had fallen from $250 \mu \mathrm{mol} / \mathrm{L}$ to $211 \mu \mathrm{mol} / \mathrm{L}$; the haemoglobin had risen, and the platelet count had normalised. Tacrolimus was not restarted. Two weeks later, both the HBV viral load and serum creatinine had further improved to $24884 \mathrm{IU} / \mathrm{mL}$ and $171 \mu \mathrm{mol} / \mathrm{L}$, respectively. Several months later, transplant function remains stable at this new baseline and HBV viral load continues to fall.

We hypothesise that the acute allograft dysfunction was secondary to an infection associated (HBV) immune complex mediated glomerulonephritis. Our patient developed acute $\mathrm{HBV}$ despite successful HBV vaccination before transplant.

\section{Discussion}

It is widely accepted that patients receiving RRT should be vaccinated against $\mathrm{HBV}$, ideally well in advance of ESKD. 




(a)

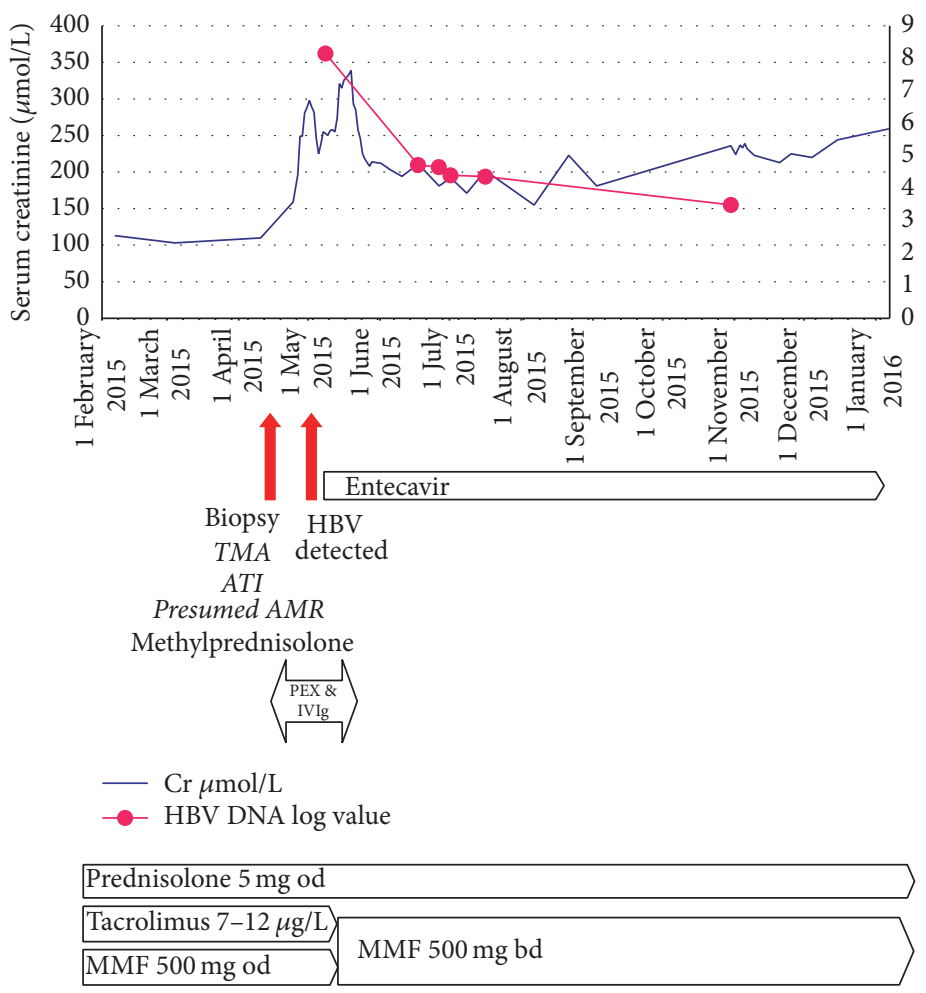

(b)

FIGURE 1: (a) Events following transplantation. (b) Events following his presentation with AKI.

Anti-HBs levels should be checked annually and boosted as indicated $[2,6,7]$.

The importance of preventing HBV infection among solid organ transplant candidates and recipients is twofold; immunosuppressed individuals are more susceptible to infection with HBV and if infected with HBV are more likely to develop chronic HBV infection. Furthermore, a transplant candidate may be offered an organ from a HBsAg-negative,
$\mathrm{HBcAb}$-positive ("core-positive") donor, so there is additional benefit to completing HBV immunisation before transplantation [8].

If a kidney transplant recipient has not been vaccinated against $\mathrm{HBV}$ prior to transplantation then current best practice is to vaccinate between 2 and 6 months after transplant, thereby avoiding the risk that high dose immunosuppression may blunt the vaccine response [9]. The response 




(a)

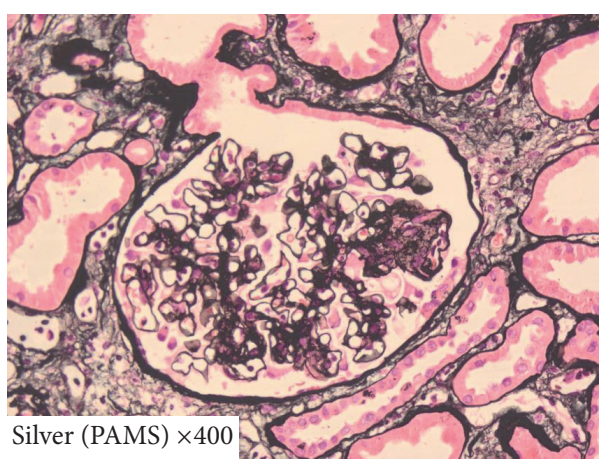

(c)

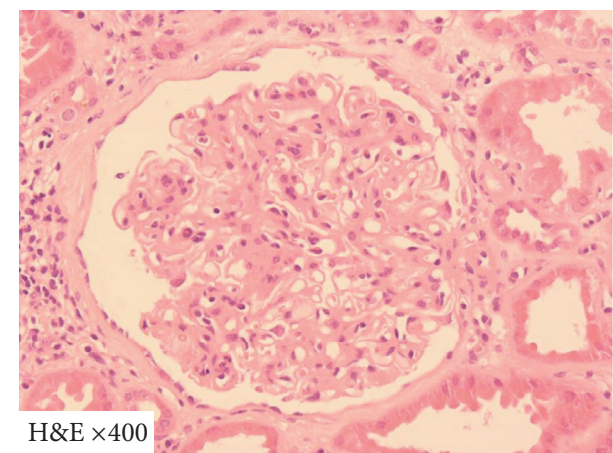

(b)

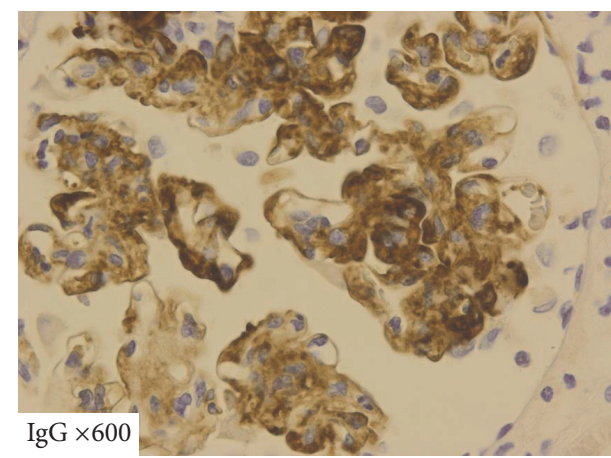

(d)

FIGURE 2: (a) Acute thrombotic occlusion of capillary loops, with segmental endocapillary hypercellularity due to inflammation, with endothelial cell swelling, and with fragmented red blood cells. (b) Mild increase in mesangial cells and moderate expansion of the mesangial matrix. Occasional inflammatory cells in capillary loops. (c) Segmental endocapillary hypercellularity with endothelial cell swelling due to inflammatory cells. (d) Positive staining for immunoglobulin G in the matrix and along glomerular capillary walls. Staining for immunoglobulins $\mathrm{A}$ and $\mathrm{M}$ and $\mathrm{C} 3$ and $\mathrm{Clq}$ was also positive.

to $\mathrm{HBV}$ vaccine administration after transplantation varies greatly, with response rates ranging between 17 and $89 \%$ [10-12]. Unfortunately, HBsAb levels decline more rapidly in immunosuppressed patients, even in those who develop protective levels $[10,13]$, and booster doses produce blunted responses [10]. These results underscore the value of pretransplant immunisation, ideally prior to the onset of advanced kidney failure.

The need for hepatitis B vaccination boosters is controversial and practice varies from country to country. There have however been reports of clinically significant $\mathrm{HBV}$ infection in previously immunised dialysis patients in whom production of HBsAb was no longer measurable [1].

It is widely accepted that annual measurement of $\mathrm{HBsAb}$ levels inform the use of booster doses of hepatitis B vaccine for dialysis patients. The European Consensus Group on hepatitis B immunity have expanded this recommendation to include patients with impaired immune function [14].

KDIGO [3] and the UK Renal Association [4] recommend $\mathrm{HBV}$ vaccination for kidney transplant recipients ideally before transplant, with an antibody check 6-12 weeks after the vaccination course. Annual antibody level checks before and after transplantation are recommended, with a booster to be given if the antibody level drops below $10 \mathrm{mIU} / \mathrm{mL}$.
Our case demonstrates that HBV can cause clinically significant disease after transplantation, even when a patient has been vaccinated against $\mathrm{HBV}$ before transplantation with apparently protective response. This case is an illustrative example of the possible benefits of following the current International and UK Renal Association guidance.

This case is also notable for the association between acute HBV infection and biopsy-proven TMA with immune complex mediated glomerulonephritis causing acute allograft dysfunction. HBV infection is associated with a range of kidney diseases, most commonly membranous nephropathy and mesangiocapillary glomerulonephritis. However, an association or even causative role for HBV in TMA and immune complex mediated glomerulonephritis has not previously been reported.

\section{Competing Interests}

The authors declare that there is no conflict of interests regarding the publication of this paper.

\section{References}

[1] CDC, "Recommendations for preventing transmission of infections among chronic hemodialysis patients," Morbidity and 
Mortality Weekly Report: Recommendations and Reports, vol. 50, pp. 1-43, 2001.

[2] Renal Association Guidelines, http://www.renal.org/guidelines/ modules/blood-borne-virus-infection\#S5.

[3] http://kdigo.org/clinical_practice_guidelines/pdf/TxpGL_publVersion.pdf.

[4] Clinical Practice Guidelines, Post-Operative Care of the Kidney Transplant Recipient, Guideline 8.1.2, UK Renal Association, 5th edition, 2011.

[5] R. L. Da Silva, "Viral-associated thrombotic microangiopathies," Hematology/Oncology and Stem Cell Therapy, vol. 4, no. 2, pp. 51-59, 2011.

[6] A. F. Charest, A. Grand'Maison, J. McDougall, and M. B. Goldstein, "Evolution of naturally acquired hepatitis B immunity in the long-term hemodialysis population," American Journal of Kidney Diseases, vol. 42, no. 6, pp. 1193-1199, 2003.

[7] I. Tsouchnikas, E. Dounousi, K. Xanthopoulou, S. Papakonstantinou, V. Thomoglou, and D. Tsakiris, "Loss of hepatitis B immunity in hemodialysis patients acquired either naturally or after vaccination," Clinical Nephrology, vol. 68 , no. 4, pp. 228234, 2007.

[8] R. T. Chung, S. Feng, and F. L. Delmonico, "Approach to the management of allograft recipients following the detection of hepatitis B virus in the prospective organ donor," American Journal of Transplantation, vol. 1, no. 2, pp. 185-191, 2001.

[9] L. G. Rubin, M. J. Levin, P. Ljungman et al., "2013 IDSA clinical practice guideline for vaccination of the immunocompromised host," Clinical Infectious Diseases, vol. 58, no. 3, pp. e44-e100, 2014.

[10] C. Loinaz, J. R. de Juanes, E. Moreno Gonzalez et al., "Hepatitis B vaccination results in 140 liver transplant recipients," Hepatogastroenterology, vol. 44, no. 13, pp. 235-238, 1997.

[11] S.-H. Chang, K.-S. Suh, N.-J. Yi et al., "Active immunization against de novo hepatitis $\mathrm{B}$ virus infection in pediatric patients after liver transplantation," Hepatology, vol. 37, no. 6, pp. 13291334, 2003.

[12] B. Serrano, J.-M. Bayas, L. Bruni, and C. Díez, "Solid organ transplantation and response to vaccination," Vaccine, vol. 25, no. 42, pp. 7331-7338, 2007.

[13] P. J. Grob, U. Binswanger, K. Zaruba et al., "Immunogenicity of a hepatitis B subunit vaccine in hemodialysis and in renal transplant recipients," Antiviral Research, vol. 3, no. 1, pp. 4352, 1983.

[14] European Consensus Group on Hepatitis B Immunity, "Are booster immunisations needed for lifelong hepatitis B immunity?" The Lancet, vol. 355, pp. 561-565, 2000. 


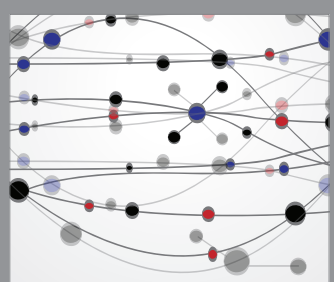

The Scientific World Journal
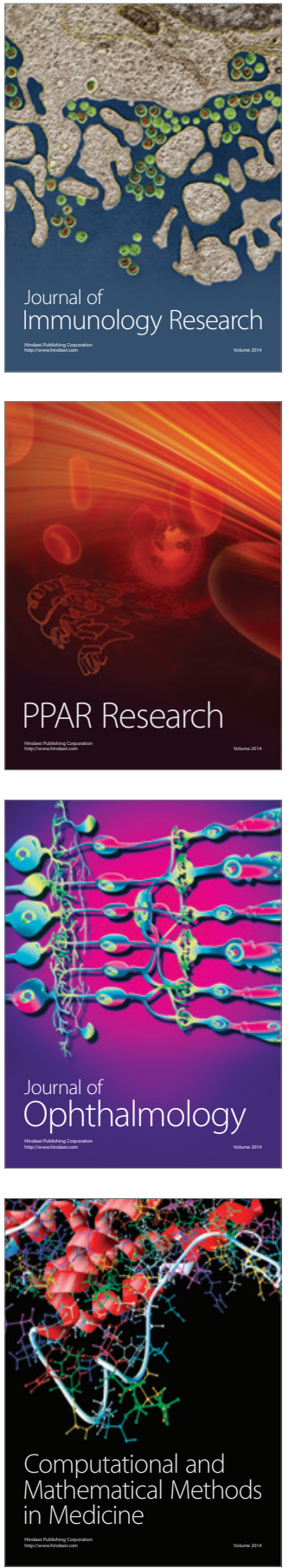

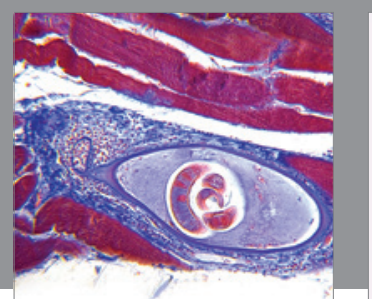

Gastroenterology Research and Practice

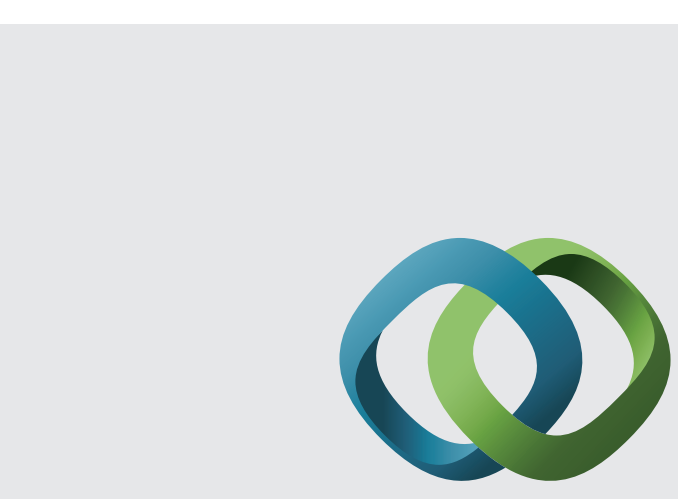

\section{Hindawi}

Submit your manuscripts at

http://www.hindawi.com
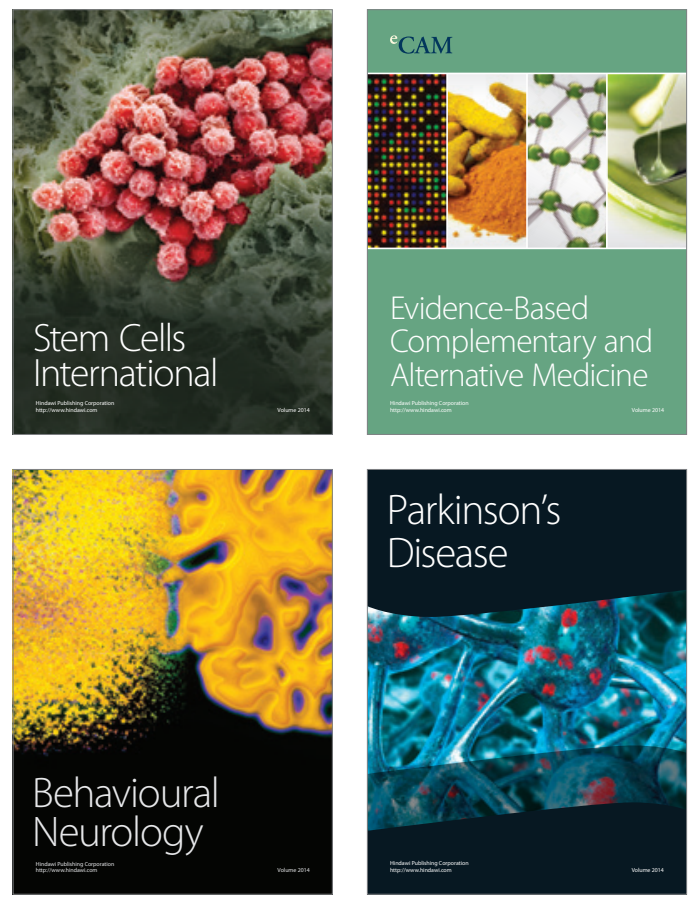


Disease Markers
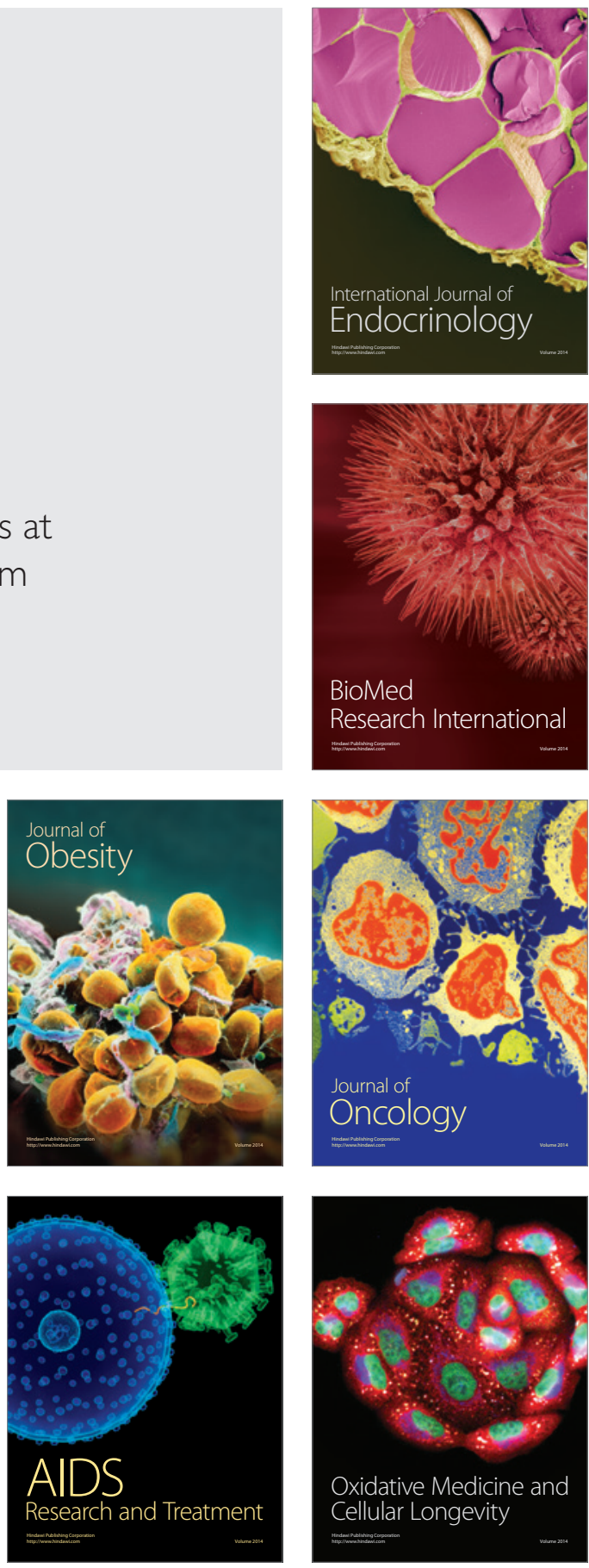